\title{
THE SUN IN SUBMILLIMETER RADIATION
}

\author{
CHARLES LINDSEY \\ Solar Physics Research Corporation, 4720 Calle Desecada, Tucson, AZ 85718, U.S.A.
}

\begin{abstract}
Continuum observations in the far IR have given us a broad spectrum of new and powerful diagnostic utilities for the solar atmosphere. The infrared continuum is formed in LTE with thermal free electrons by free-free interactions. This gives us a flexible and accurate atmospheric thermometer that has made infrared measurements fundamental to modeling of the quiet solar medium for more than two decades. The submillimeter and millimeter continua are particularly useful with respect to thermal diagnostics of the low chromospheric temperature minimum, where non-radiative heating of the solar medium becomes clearly manifest. Modern submillimeter telescopes and instrumentation on Mauna Kea, in Hawaii, are now revolutionizing solar observations in the submillimeter spectrum, giving us the first observations of detail on the scale of the chromospheric supergranular network, sunspots and prominences. These observations are showing us a remarkable and unexpected view of thermal structure that emerges as one probes to successively higher levels above the chromospheric temperature minimum.
\end{abstract}

Key words: heating - infrared: stars - Sun: chromosphere - Sun: filaments - sunspots

\section{Introduction}

The submillimeter spectrum is clearly defined as excluding wavelengths longward of $1 \mathrm{~mm}$, but its short-wavelength limit is somewhat arbitrary. The terrestrial atmosphere blocks almost all radiation between 30 and $300 \mu \mathrm{m}$, (see Figure 2 of Jefferies, 1993, this volume). This occlusion seems to provide a rough discriminator for astronomers whereby $300 \mu \mathrm{m}$ is definitely considered submillimeter while $30 \mu \mathrm{m}$ falls in the far-infrared domain and is rarely thought of as submillimeter. However, the $30-300 \mu \mathrm{m}$ band is observable from the stratosphere or above, and is of great interest in solar diagnostics. We will consider wavelengths greater than $30 \mu \mathrm{m}$ to be submillimeter, for the purpose of this discussion, but a clear boundary is actually not established.

The submillimeter solar spectrum, at first sight, appears to exude a certain blandness. As wavelength increases, spectral lines weaken leaving only a few diffuse emission lines longward of $100 \mu \mathrm{m}$ (e.g., Boreiko and Clark 1986; Naylor et al. 1993 find no lines longward of $300 \mu \mathrm{m}$ ). The continuum offers spatial information, but at a high cost, for diffraction reduces spatial resolution. Nevertheless, the submillimeter spectrum does present us with an exceptionally powerful diagnostic perspective. It has served a unique role as a thermal probe of the chromospheric medium for the quiet Sun. And now, modern large submillimeter telescopes are opening this diagnostic to a broad range of other interesting chromospheric structure. A brief review of the mechanisms important in submillimeter radiative transfer in the solar atmosphere offers some insight into this role.

\section{Continuum Emission and Absorption}

The primary mechanism of continuum emission, and thus also opacity, throughout of the infrared spectrum longward of $2 \mu \mathrm{m}$ is collisions of free electrons with atoms and ions, i.e., bremsstrahlung. For collisions with neutral hydrogen atoms, the 


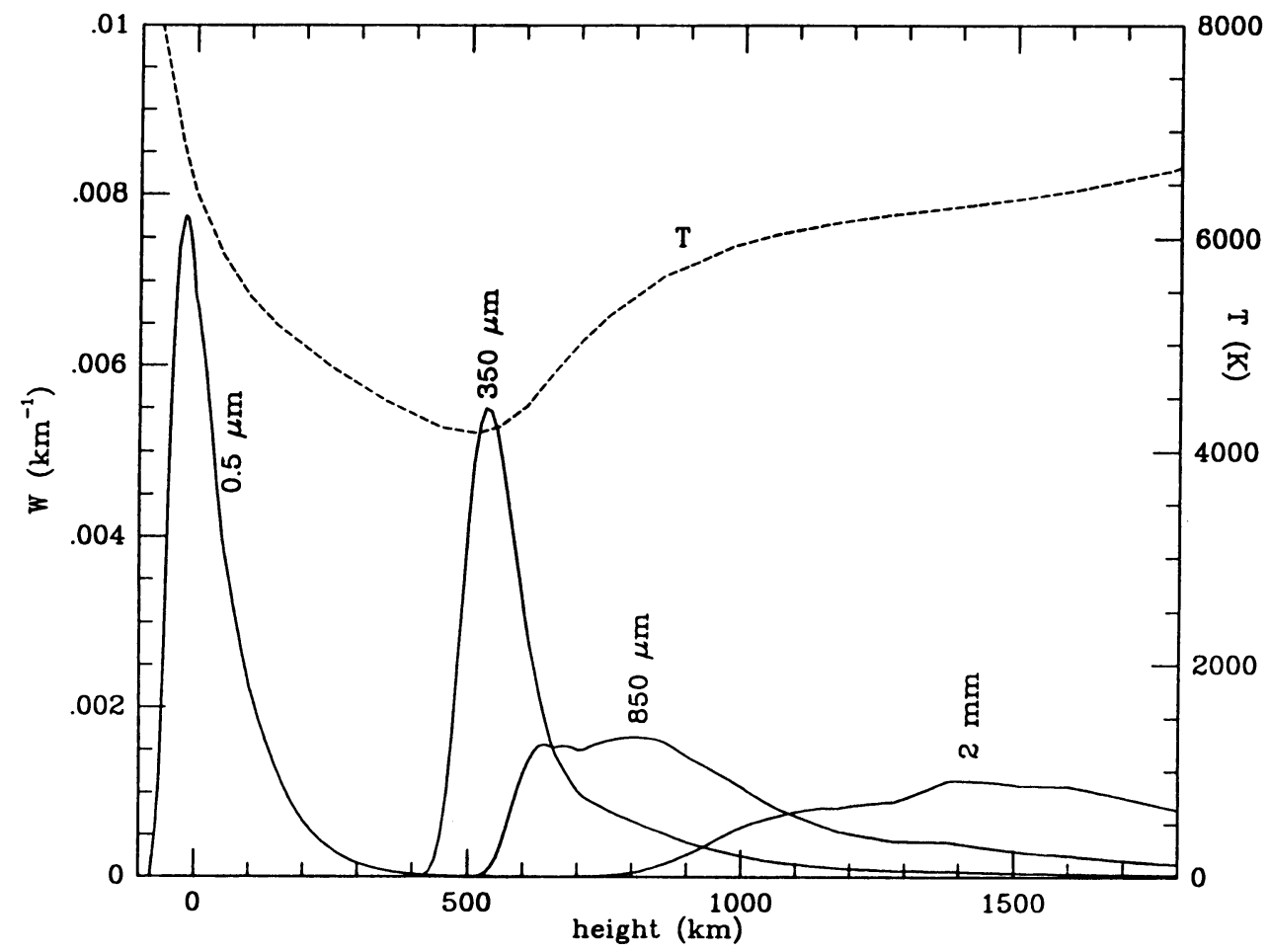

Fig. 1. Weighting functions for 350,850 and $2,000-\mu \mathrm{m}$ continuum radiation are plotted against the temperature profile (dashed curve) of model C of VAL III.

process is termed $\mathrm{H}^{-}$free-free emission, after a formalism that considers the free electron together with the neutral atom as an unbound $\mathrm{H}^{-}$ion both before and after the interaction. $\mathrm{H}^{0}$ free-free emission, i.e., electrons colliding with protons (or any other singly charged ion), begins to dominate $\mathrm{H}^{-}$free-free emission when the atmospheric medium is more than about a half percent ionized. Free-free opacity increases strongly with wavelength, $\lambda$, in the infrared. For $\mathrm{H}^{-}$free-free opacity, it is proportional to $\lambda^{2}$, and this rule also serves as a rough approximation for $\mathrm{H}^{0}$ freefree opacity. Figure 1 shows weighting functions for 350,850 and 2,000 $\mu$ m radiation for atmospheric model C of Vernazza, Avrett and Loeser 1981 (VAL III C). The shorter wavelengths, centered in atmospheric windows accessible from Mauna Kea, are formed by chromospheric levels at which the temperature begins to increase with height.

Because the emission arises from thermal collisions, the infrared continuum is formed essentially in thermal equilibrium with the local thermal free electrons that give rise to it. The source function is the Planck function, which at solar temperatures follows the Rayleigh-Jeans law and is therefore simply proportional to temperature. It is useful to keep in mind that in a non-uniform medium the visible and ultraviolet Planck functions at solar temperatures are weighted strongly 
toward hotter elements, so much so that images at the shorter wavelengths may represent mostly a rather small fraction of the very hottest gas. In the infrared, one can usually suppose that the continuum intensity roughly represents the temperature of gas at about unit optical depth, rather than optically thin gas that has a disproportionately large source function. All of these qualities make the infrared and submillimeter continuum surely the best known chromospheric thermometer.

\section{Recent Submillimeter Observations}

I will bypass a large amount of interesting past work at submillimeter wavelengths to look over a sample of recent exciting results from the world's largest submillimeter telescope, the 15-m James Clerk Maxwell Telescope (JCMT) on Mauna Kea. Figure 3 of Jefferies (1993) shows an image covering most of the solar disk in $850 \mu \mathrm{m}$ radiation (Panel $a$ ) next to a simultaneous image of the Sun in the K-line of singly ionized calcium (Panel $b$ ). There are striking similarities. Both images clearly show the chromospheric supergranular network. In this Figure, the submillimeter contrast (Rayleigh-Jeans) has been enhanced to match the greater K-line contrast. Lindsey and Jefferies (1991a) find the distribution of infrared intensities from the supergranular network to be consistent with that expected from the VAL III (1981) models.

Notwithstanding clear similarities, there are some clear differences between the $850 \mu \mathrm{m}$ image and the $\mathrm{K}$-line image: In particular, the $\mathrm{K}$-line image is strongly limb darkened, where the $850 \mu \mathrm{m}$ image is slightly limb brightened, a quality first inferred observationally by Noyes, Beckers and Low (1968) and mainly attributable to a general increase in chromospheric temperature with height. The extreme limb brightnesses measured by occultation during eclipse are reviewed by Clark (1993) in this volume. (For a summary glance see Figure 4 of Jefferies 1993, also in this volume). The strong limb-darkening seen in the K-line reflects an increasing departure of the K-line source function from local thermal equilibrium (LTE) with increasing height, as decreasing opacity allows the calcium ions to radiate more efficiently into space. This is one of the stronger of the non-LTE manifestations that are shared by all chromospheric lines in the visible, and we now know that at least some photospheric lines far into infrared depart from LTE (see Chang 1993 and Rutten and Carlson 1993, in this volume).

For a smooth atmosphere, there is a simple formalism relating the infrared continuum limb brightness profile to the vertical temperature gradient and similarly to the disk-center temperature as a function of wavelength. Where the $\lambda^{2}$ opacity rule is accurate, for instance, the brightness temperature, $T_{b}$, of radiation emerging at incidence $\theta$ is simply a function of the product $\lambda^{2} / \mu$ :

$$
T_{b}(\lambda, \mu)=f\left(\lambda^{2} / \mu\right),
$$

where $\mu=\cos \theta$ (Simon and Zirin, 1969). These relationships had already lead to predictions of strong limb brightening in radio measurements in the 1950s, an effect that never clearly materialized for wavelengths of order $1 \mathrm{~cm}$ or greater. Equation (1) is not satisfied because the chromosphere is not smooth. This realization lead to models incorporating rough structure such as spicules as early as Coates (1958). 
a

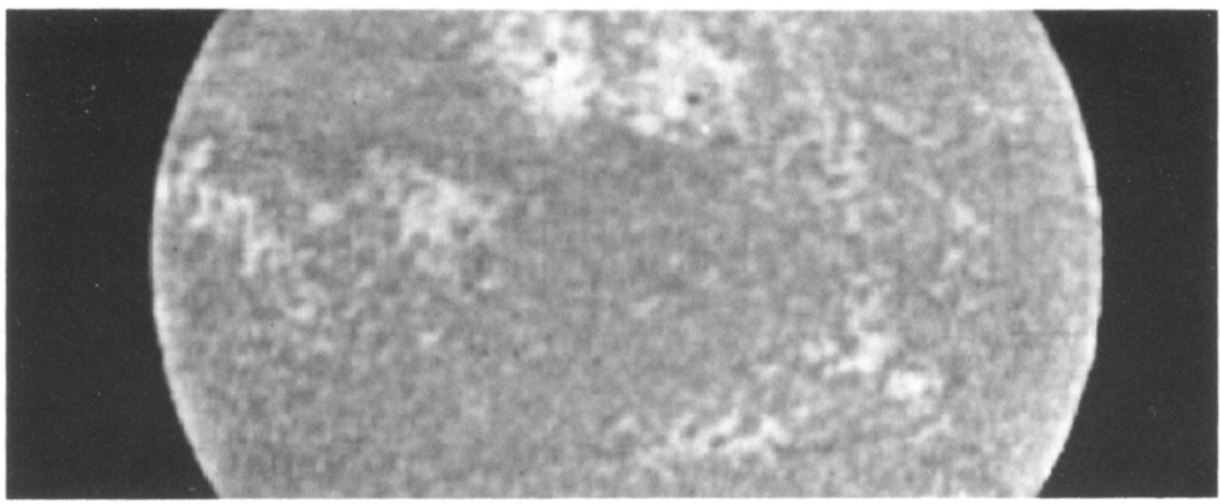

b

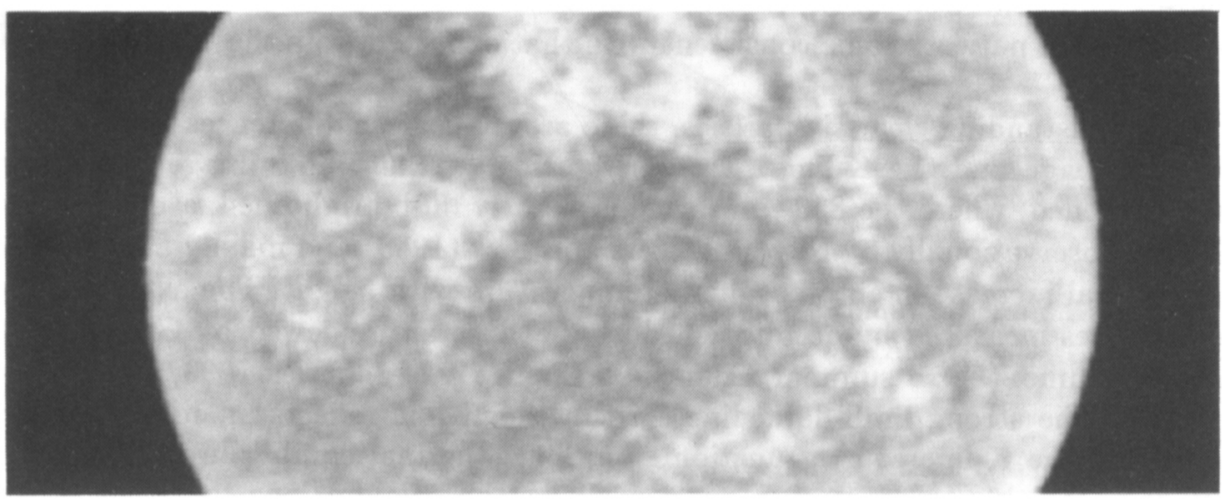

Fig. 2. Images of the Sun in $350-\mu \mathrm{m}$ (Panel $a$ ) and $1,200 \mu \mathrm{m}$ radiation (Panel $b$ ) made on $1991 \mathrm{Feb} 9$ at $\sim 2200$ UT and $\sim 2500$ UT respectively. Vertical and horizontal fiducials indicate the positions of large sunspots.

The best available review of the limb-brightness problem remains that of Simon and Zirin (1969). The submillimeter continuum seems to be unique in exhibiting clear limb brightening.

An indication of how heated chromospheric structure emerges above the temperature minimum level is found in Figure 2, which shows images of the Sun in 350and $1200-\mu \mathrm{m}$ radiation taken a few hours apart. Network boundaries that appear relatively faint and thin at $350 \mu \mathrm{m}$ brighten and appear to expand to a greater filling in $1200-\mu \mathrm{m}$ radiation. However, at least some part of this expansion is attributable to diffraction. 


\subsection{Sunspots}

Vertical and horizontal fiducials in Figure 2 show the locations of two large sunspots. They appear nearly invisible at these wavelengths, distinguishable from the quiet Sun only by contrast with surrounding plage. Even large sunspots appear mostly just by contrast against surrounding brighter plage. Occasionally they are as bright as the plage themselves, as seen in Figure 3 of Jefferies (1993) in this volume, where a group of spots registers as very dark in the K-line (lower right of Panel $b$ ) but resembles bright plage in the $850 \mu \mathrm{m}$ continuum (lower right of Panel $a$ ). This comparison further illustrates the drastic departure from LTE of visible chromospheric lines, which generally register large sunspots as quite dark. The best known sunspot models have low chromospheres considerably cooler than the quiet Sun temperature minimum, resulting in a dark submillimeter sunspot. The models that come closest to satisfying the submillimeter observations seem to be those of Maltby et al. (1993). It now seems that even these models will need a considerably hotter lower chromospheres to fit the new submillimeter and millimeter observations, requiring a strong heating mechanism in those regions with the most intense magnetic fields.

\subsection{LARge-Scale VARIations IN The QUiET SUN}

While the $850 \mu \mathrm{m}$ images clearly show the quiet-Sun supergranular network (see Figure 3 of Jefferies, 1993, in this volume), they also show large-scale quiet-Sun intensity variations that the $\mathrm{K}$-line does not. There are extended regions up to $10 \%$ darker than the more typical quiet Sun, and these seem to have a weak tendency to border active regions. They are not apparent at $350 \mu \mathrm{m}$ (Fig. $2 a$ ), but are pronounced at $1,200 \mu \mathrm{m}$ (Fig. $2 b$ ). One is tempted to think of such a region as a sort of chromospheric hole. However, preliminary estimates indicate that even these darkened regions have a greater brightness temperature in 1,200 $\mu$ m radiation than the quiet Sun in $350 \mu \mathrm{m}$ radiation, thus, they evidently are not "chromospheric holes," but perhaps simply regions of reduced heating. While not seen in the calcium Kline, similar features are clearly seen in the calcium infrared triplet and weakly in the sodium D-lines, where large active regions tend to be surrounded by darkened halos.

\subsection{Filaments and Prominences}

Filaments seem to be nearly invisible against the solar disk at submillimeter wavelengths. This strongly suggests material roughly equal in temperature to the low chromosphere, $\sim 5,500 \mathrm{~K}$, which is considerably cooler than most prominence models but not all (see, e.g., Hirayama 1985 and Hirayama, Nakagomi and Okmoto 1979). We know that filaments have a substantial optical depth in the submillimeter continuum, because they are clearly seen as prominences in this radiation. Kosugi Ishiguro and Kiyoto (1986) clearly see filaments as dark against the solar disk at $3 \mathrm{~mm}$, where the disk brightness is considerably higher than $5,500 \mathrm{~K}$. Thus, submillimeter and radio observations are accumulating a strong case in favor of prominences being of mostly relatively cool material. 


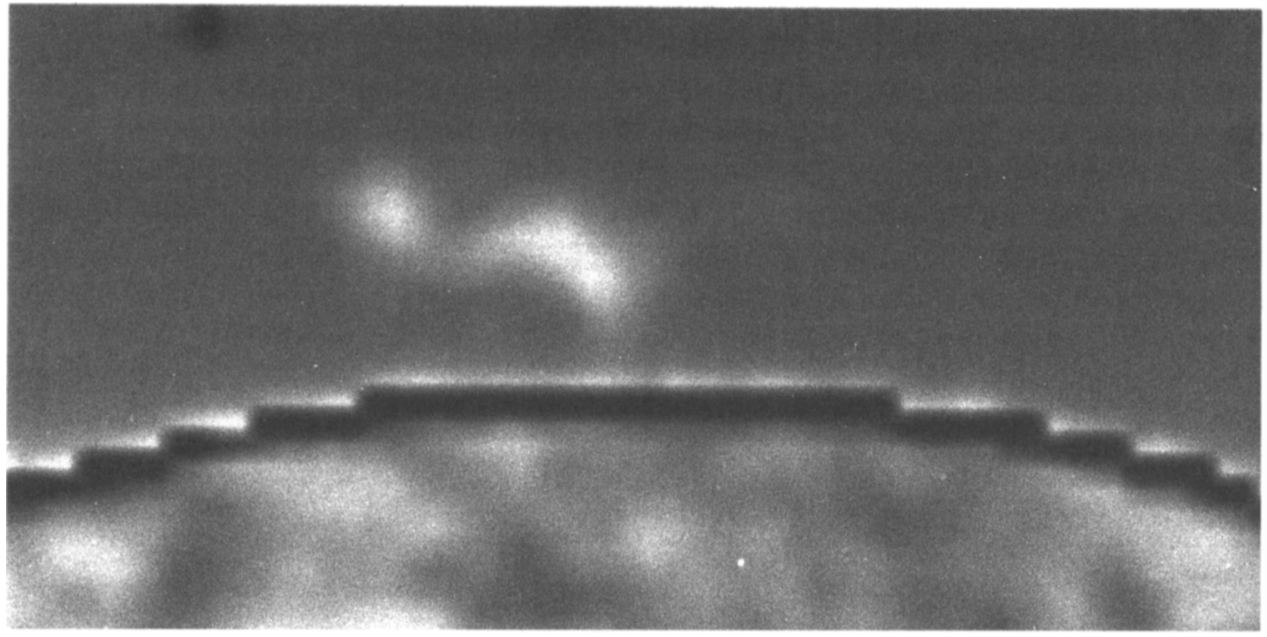

Fig. 3. A large active prominence in $1,300 \mu \mathrm{m}$ radiation on 1991 July 10 at $1800 \mathrm{UT}$. The prominence was raster-scanned by the $15 \mathrm{~m} \mathrm{JCMT} 23 \mathrm{hr}$ before a total solar eclipse over the facility on Mauna Kea.

Figure 3 shows a large prominence in 1.3-mm radiation. This prominence shows a peak brightness temperature of $\sim 2,500 \mathrm{~K}$. Rough estimates, assuming an optical path length through the prominence comparable to its horizontal dimensions and a temperature of $5,500 \mathrm{~K}$ suggest a free electron density of order $10^{10} \mathrm{~cm}^{-3}$ (Harrison et al. 1993).

\subsection{Chromospheric Dynamics}

Infrared thermal diagnostics are as applicable to chromospheric dynamics as to quasi-static modeling. Kaufmann et al. (1993) and Correia et al. (1993) explain the need for submillimeter observations in solar flare diagnostics. Observations even down to ms time scales are desired. The practical problem reduces to dedicating a substantial submillimeter facility to a program to monitor active regions for long enough to get a good sampling of large-flare observations.

Far infrared diagnostics have already given us very interesting thermal diagnostics of the response of the chromospheric medium to compressional perturbations due to the five-minute oscillations in the quiet Sun. Figure 4, taken from Kopp et al. (1992), shows time series of local brightness oscillations measured at 50, 100 and $200 \mu \mathrm{m}$ from the Kuiper Airborne Observatory, made simultaneously with Doppler observations of the same region in the sodium $\mathrm{D}_{1}$ line from the Stokes Polarimeter at the Mees Solar Observatory (see Mickey 1985) on 1987 May 14. There is a high correlation between velocity and submillimeter intensity, with intensities leading velocity by varying phase shifts. Observations in visible lines, particularly those of Lites, Chipman and White (1982) have shown similar phase shifts between velocity and core brightness of certain members of the calcium infrared triplet, particularly Ca II $8498 \AA$. Kopp et al. 1992 use the measured infrared phase-shifts to 


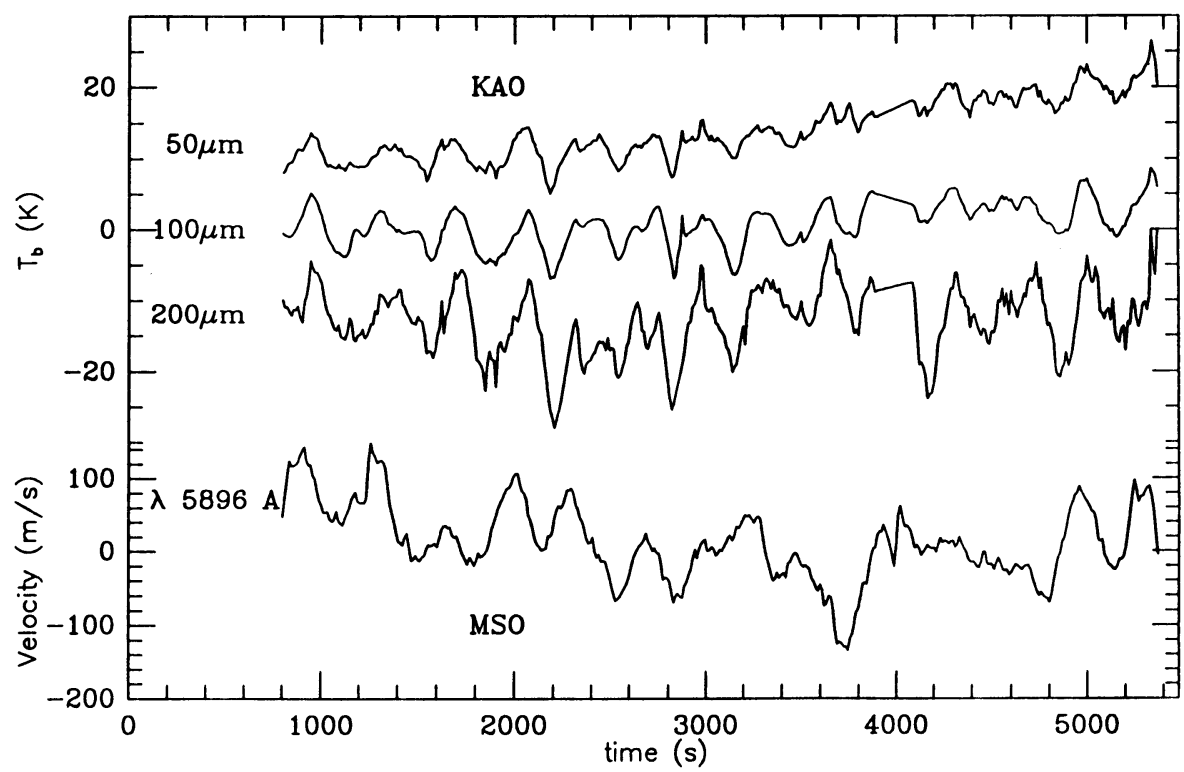

Fig. 4. Time-series showing brightness variations in 50, 100 and $200 \mu \mathrm{m}$ continua (top three plots) in response to five-minute oscillations monitored by Doppler variations in the Sodium $\mathrm{D}_{1}$ line $\lambda 5896 \AA$ (bottom plot).

characterize the low chromosphere by thermal relaxation at various rates over a range of heights spanning the temperature minimum. They find relaxation rates that decrease rapidly with height, suggesting a simple adiabatic response of the chromospheric medium above about $1,000 \mathrm{~km}$. In the low chromosphere, these rates appeared unexpectedly large at the time the observations were made, but now seem reasonably consistent with recent theoretical work by Anderson (1992). Again, the interpretation of the infrared continuum is greatly simplified by the emission being always in LTE with the thermal free electrons.

\section{Summary}

The infrared continuum remains central to thermal diagnostics of the solar photosphere and chromosphere. Modern large submillimeter telescopes and instrumentation are opening a broad new range of solar phenomena to submillimeter observations, and are opening us to a remarkable, new perspective on chromospheric structure. The LTE source function of the infrared continuum promises to facilitate our interpretation of the infrared in terms of atmospheric models, especially where rough, inhomogeneous atmospheric structure is important, as it is in the chromosphere (see Lindsey and Jefferies, 1991b).

With more advanced instrumentation now being developed, we can expect submillimeter observations to give us considerably more insight into dynamical qualities 
of the chromosphere in its response to acoustic and other hydro-mechanical perturbations, such as the five-minute oscillations, and to transient events, such as flares. Even the observation of a clear lack of submillimeter emission from a moderately large flare would be interesting (see Kaufman 1993). Good flare observations in this region are problematical for the moment, but modern array detectors now being developed will soon make them practical. It seems clear that we have only obtained a first glimpse of the potential of submillimeter diagnostics at this juncture.

\section{Acknowledgements}

This review presents original results from the James Clerk Maxwell Telescope, run by the Joint Astronomy Center, based at the University of Edinburgh. This work was also supported by NSF Grant ATM-9122073. The author made extensive use of facilities at the National Solar Observatory, where he holds a visiting appointment.

\section{References}

Anderson L.: 1992, private communication.

Boreiko, R.T. and Clark, T.A.: 1986, Astron. Astrophys., 157, 353.

Chang, E. S.: 1993, these proceedings.

Clark, T. A.: 1993, these proceedings.

Coates, R. J.: 1958, Astrophys. J. 128, 83.

Correia, E., Kaufmann, P. and Magnum, A.: 1993, these proceedings.

Harrison, R. A., Carter, M. K., Clark, T. A., Lindsey, C., Jefferies, J. T., Sime, D. G., Watt, G., Roellig, T. L., Becklin, E. E., Naylor, D. A. , Tomkins, G. J. and Braun, D. C.: 1993, Astron. Astrophys., in press.

Jefferies, J. T.: 1993, these proceedings.

Hirayama, T.: 1985, Solar Phys., 100, 413.

Hirayama, T., Nakagomi, Y. and Okmoto, T.: 1979 in E. Jensen, P. Maltby, and F. Q. Orrall (eds.), 'Physics of Solar Prominences', Proc. IAU Colloq. 44, 48.

Kaufman, P., Correia, E., Costa J. E. R. and Zodi, A. M.: 1993, these proceedings.

Kopp, G., Lindsey, C., Roellig, T. L., Werner, M. W., Becklin, E. E., Orrall, F. Q., Jefferies, J. T.: 1992, Astrophys. J. 388, 203.

Kosugi, T., Ishiguro, M. and Kiyoto, S. K.: 1986, Pub. Astr. Soc. Japan, $38,1$.

Lindsey, C. and Jefferies, J. T.: 1991(a), A strophys. J., 349, 286. (radiative transfer).

Lindsey C. and Jefferies, J. T.: 1991(b), Astrophys. J., 383, 443. (chromospheric supergranular net work).

Lites, B. W., Chipman, E. G. and White, O. R.: 1982, Astrophys. J., 253, 367.

Maltby, P.: 1993, these proceedings.

Mickey, D. L.: 1985, Solar Phys., 97, 223.

Naylor, D. A.: 1993, these proceedings.

Noyes, R. W., Beckers, J. M. and Low, F. J.: 1968, Solar Phys. 3, 36.

Rutten R. and Carlsson, M.: 1993, these proceedings.

Simon, M. and Zirin, H.: 1969, Solar Phys., 9, 317.

Vernazza, J. E., Avrett, E. H., and Loeser, R.: 1981, Astrophys. J. Supp., 45, 635. 